\title{
Low complex interference cancellation via modified suboptimum search algorithm and reduced rank linear detection for mobile uplink
}

\author{
M. Mozaffaripour \\ Mobile Group, CCSR, Electronics Engineering \\ University of Surrey \\ Guildford, UK \\ m mozaffari2001@yahoo.co.uk
}

\author{
R.Tafazolli \\ Mobile Group, CCSR, Electronics Engineering \\ University of Surrey \\ Guildford, UK \\ R.Tafazolli@surrey.ac.uk
}

\begin{abstract}
A Low complex interference cancellation via modified suboptimum search algorithm in conjunction with a primary stage of reduced rank linear (RRL) multiuser detector for the mobile uplink is proposed. Initial stage is improved through mathematical analysis via Gershgorin algorithm in linear algebra and RRLG detector is introduced. The complexity of the initial stage is evaluated and compared to the recently reported low-complex Fourier interference cancellation method. Depending on the value of the spreading factor of the active users in system, RRLG outperforms the Fourier algorithm, in terms of complexity. The structure of the RRLG method and the suboptimum search algorithm are well suited together and makes them collaboratively work without encountering a high level of complexity. Considering the power profile of the users in the suboptimum search algorithm leaded to even less complexity, yet keeping the performance almost the same. The performance of the structure is obtained via simulations and has been compared to partial parallel interference cancellation (PPIC) method. A good improvement in performance in the low SNR regions, which is difficult to achieve by conventional multiuser detectors and also important as the actual systems are likely to operate in these regions, has been achieved. All the techniques and their modifications introduced in this work consider the complexity as an important issue that enables them suitable for industry and implementation purposes. Another important feature is that the techniques perform on canonical matrix formulations of the system so they can be applied to MC-CDMA and MIMO systems, as well.
\end{abstract}

Keywords- Terms- suboptimum search algorithm, Polynomial expanded linear multiuser detection, CDMA

\section{INTRODUCTION}

Conventional detection in CDMA systems, composed of a bank of matched filters, suffers from the Multiple Access Interference (MAI). Several suboptimum multiuser detectors have been proposed for mitigating. Amongst them are linear detectors, like Decorrelator and MMSE. These methods have several attractive properties but suffer from implementation complexity, which is mainly due to a need for inverting a large matrix. Some works are done for approximating the inverse matrix without computing directly [1-3]. For example [3] uses polynomial expansion (PE) method. This method is powerful, however needs to calculate a set of coefficients. On the other hand [1] has proposed a simplified polynomial-expansion, which according to its inaccurate parameter estimation, the convergence rate is rather slow. There is another issue about linear suboptimum algorithms that there is still a gap between their performance and the optimum detector. Optimum detector relies on exhaustive searches and hence impractical. Designing suboptimum search strategies with moderate level of complexity can fill this gap. This paper tackles the above issues by proposing a low complex structure composed of modified suboptimum search algorithm in conjunction with a reduced rank linear (RRL) multiuser detector without a need to matrix inversion. We first briefly review the system model of CDMA systems in section II. In section III RRL detector is addressed. Section IV considers the complexity evaluation of the RRL and the Fourier algorithm. In section $\mathrm{V}$, the suboptimum search algorithm operating on the output of both conventional and RRLG detector is introduced. Power profile of the users in the proposed scheme is also considered and a more simple structure with the almost same performance is achieved. The results are given in section VI. Conclusions are summarized in section VII.

\section{SYSTEM MODEL}

First of all a model for the CDMA systems is needed. The received signal for a system with $\mathrm{K}$ users is:

$$
r(t)=\sum_{k=1}^{K} s_{k}\left(t-\tau_{k}\right)+n(t)
$$

where $n(t)$ is the single source of the channel and $s_{k}(t)$ is the received signal for the user $k$ and is defined as follow

$$
s_{k}(t)=\sqrt{P_{k}} a_{k}(t) b_{k}(t) e^{j \theta_{k}}
$$

$P_{k}, a_{k}(t)$ and $b_{k}(t)$ are $k^{\text {th }}$ user's power, spreading waveform and data waveform respectively. (Both waveforms are assumed to be rectangular pulses). $\theta_{k}$ is the received phase of the $k^{\text {th }}$ user relative to some reference phase.

After passing through a bank of matched filters the output of the filters can be shown to be as follows:

$$
\mathbf{y}=\mathbf{R A b}+\mathbf{n}
$$

where $\mathbf{R}$ is a matrix with the size of $K N_{b} \times K N_{b}$ and relates to cross-correlation of the spreading waveforms. $N_{b}$ is the number of bits considered for processing. $\mathbf{A}$ is a 
$K N_{b} \times K N_{b}$ diagonal matrix with square roots of the received signals' energies.

\section{Reduced rank linear (RRL) multiuser detector using Taylor series and Gershgorin algorithm}

\section{A) Previous research}

By using the Taylor series, the expansion of $\mathbf{R}^{-1}$ can be written as $[4,5]$ :

$$
\alpha^{-1} \mathbf{R}^{-1}=\sum_{i=0}^{\infty}(\mathbf{I}-\alpha \mathbf{R})^{i}
$$

If and only if the eigen-values of $\mathbf{R}$ satisfy the conditions:

$$
\left|1-\lambda_{i}(\alpha \mathbf{R})\right|<1
$$

For a positive subdefinite matrix $\mathbf{R}$, the above condition can be written as:

$$
0<\alpha<\frac{2}{\lambda_{\max }(\mathbf{R})}
$$

All of the eigenvalues of $\mathbf{R}$ are positive. Based on this fact, Ref. [1] has derived an approximation for $\alpha$

$$
\alpha=\frac{2}{\operatorname{trace}(\mathbf{R})}
$$

Because:

$$
\sum_{i} \lambda_{i}=\sum_{i} R_{i i}=\operatorname{trace}(\mathbf{R})>\lambda_{\max }(\mathbf{R})
$$

Though it is not an accurate estimate.

\section{B) Estimation of parameters based on Rayleigh-Ritz Theorem}

In a general case for the equal power and synchronous users, diagonal elements of $\mathbf{R}$ are all $1 \mathrm{~s}$ and the nondiagonal elements are in the range of, $(-\beta,+\beta), 0<\beta<1,(\beta$ is the maximum value of the cross-correlations). In [4] we derived a more precise formula for $\alpha$

$$
\alpha=\frac{2}{1+\beta(K-1)}
$$

In situation when the cross-correlation values are large, $\beta$ takes the maximum value of $(\beta=1)$ and the Eq.(9) becomes equal to Eq.(8), because:

$$
\operatorname{trace}(\mathbf{R})=\sum_{i=1}^{K} R_{i i}=K
$$

Eq.(10) gives a more accurate estimate for $\alpha$ than the one obtained from the Eq.(7) and in consequence a faster convergence can be achieved.

\section{C) Asymptotic approach}

One of the recent and comprehensive methods for tackling the issue of calculating the coefficients is addressed in [6], that optimises the coefficients by signal to noise ratio aspects and uses the features of random matrixes and hence they do not encounter the eigenvalues problem. In this approach one of the key assumptions is randomness of the cross-correlation matrix's elements. This assumption does not use any more information about the elements and hence leads to some inaccuracies. Since the approach is using the random matrix theories and their theory does not include the sparse matrixes, their second assumption is that the users are synchronous because in the asynchronous scenarios the matrices are sparse. These two assumptions confine the applicability of their method in the actual systems. The main advantage of their method, however, is that the optimum asymptotic weighting coefficients for the early stages of operation are obtained in the closed form.

\section{D) Gershgorin Algorithm}

In asynchronous systems most of the elements of $\mathbf{R}$ are zero while Eq.(9) considers only the maximum value of nondiagonal elements of $\mathbf{R}$ and ignores the majority of elements that are zero. This ignorance decreases the accuracy and shows its effect in the convergence rate and the performance. To overcome this problem, we refer to the Gershgorin theorem in linear algebra [5]. According to this theorem, any eigenvalue of a matrix is located in one of the closed discs of the complex plane centred at $a_{i i}$ and having radius $\sum_{j, j \neq i}\left|a_{i j}\right|$. In other words,

$$
\left|\lambda_{\mathrm{i}}-a_{i i}\right| \leq \sum_{j, j \neq i}\left|a_{i j}\right|
$$

By a simple calculation on the elements of $\mathbf{R}$, two approximate values can be derived for $\lambda_{\min }(\mathbf{R})$ and $\lambda_{\max }(\mathbf{R})$ :

$$
\begin{gathered}
\lambda_{\max } \leq \max \left\{a_{i i}+\sum_{j, j \neq i}\left|a_{i j}\right|\right\} ; i, j=1,2, \ldots, K N_{b} \\
\lambda_{\min } \leq \min \left\{a_{i i}+\sum_{j, j \neq i}\left|a_{i j}\right|\right\}
\end{gathered}
$$

The optimum value of $\alpha$ to get the fastest convergence depends on $\lambda_{\min }(\mathbf{R})$ and $\lambda_{\max }(\mathbf{R})$ through the following equation [9]:

$$
\alpha_{\text {opt }}=\frac{2}{\lambda_{\max }+\lambda_{\min }}
$$

Using Eq.(11, 12, 13), a proper estimate for $\alpha$ could be derived.

A comparison between the performances of the above three methods is given in Fig(3).

\section{Complexity EVAluation of THE RRL AND THE FOURIER ALGORITHM}

There are other methods that have recently been reported in the literature concerning linear interference cancellation. [7] has introduced a method that performs the interference suppression in the frequency domain. They also compare their method with three other methods (which are based on Cholesky factorisation, the Levinston algorithm and the Schur algorithm) in terms of performance and complexity. The authors of [7] have also provided a program [8] that calculates complexity of their algorithms. Their main conclusion about the Fourier algorithm was that it benefits 
from a low complexity in terms of floating point operations, yet achieves the same performance as the exact Cholesky algorithm.

Throughout [7], it has been assumed that the spreading factor of the users are equal to 16 and all the complexity evaluations have been carried out based on this value. In the FDD-WCDMA uplink, the spreading factor of the users is in the range of 4-256. We will see in the next section that for high values of the spreading factors, the Fourier algorithm will suffer in complexity comparisons.

The details of the complexity evaluations are provided in [9].

It should be pointed-out that in the actual systems the matched filtering process would have already been done by other subsystems and we can apply our RRL algorithm to the output of matched filters (y) so in the complexity evaluation of RRLG-MUD two cases are considered:

1) Performing the calculations considering the complexity of matched filtering (with $\mathbf{y}$ ).

2) Performing the calculations without considering the complexity of matched filtering (without $\mathbf{y}[\mathrm{wo} / \mathrm{y}]$ ).

\section{SUBOPTIMUM SEARCH ALGORITHM}

Optimum multiuser detection needs a huge number of searches. In the jointly optimum decision, the problem is to find the $\mathbf{b}$, in such a way to maximize [2]:

$$
\Omega(\mathbf{b})=2 \mathbf{b}^{T} \mathbf{A y}-\mathbf{b}^{T} \mathbf{H b}
$$

Where $\mathbf{H}$ is un-normalized cross-correlation matrix: H=ARA. The complexity would be exponential in the product $K\left(2 \mathrm{~N}_{\mathrm{b}}+1\right)$.

\section{A) Sub optimum search algorithm}

Our suboptimum search algorithm is based on an intuitive behaviour of the primary stage of detection process, this search uses a sequential strategy.

If the search process starts off from a fair reliable starting point, then the optimum sequence will be in a close neighbourhood of the starting point and only few bits in the observation window are in error. Our strategy is to pinpoint these bits by an algorithm. Considering the sequence $\left(b_{1}, b_{2}\right.$, $\left.\ldots, b_{K}\right)$ to be the output of the initial stage, based on $\operatorname{Eq}(14)$, two values for $\Omega$ related to $\left(b_{1}, b_{2}, \ldots, b_{K}\right)$ and $\left(-b_{1}, b_{2}, \ldots\right.$, $b_{K}$ ) will be calculated. Among of these two sequences, the one with greater value for $\Omega$ will be kept as the more likely sequence and the process continues the same way for the next bit. For the second bit, two sequences of $\left(b_{1}, b_{2}, \ldots, b_{K}\right)$ and $\left(b_{1},-b_{2}, \ldots, b_{K}\right)$ will be in consideration. The length of this process is only dependent on the observation window and the number of users.

\section{B) Modified sub optimum search algorithm}

To achieve a balance between the complexity and performance, we can confine the sub optimum search to a percentage of the users. In this case, after an initial preprocessing of the users and sorting them based on their

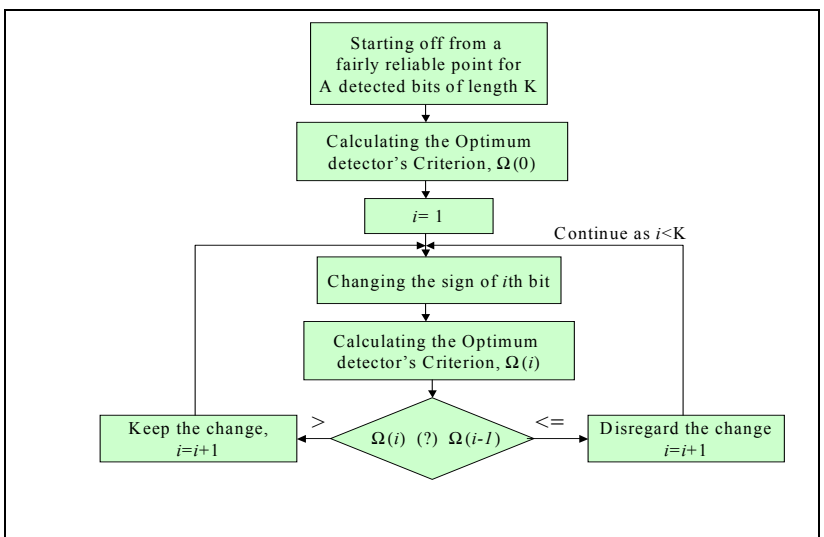

Figure 1. The sub optimum search algorithm

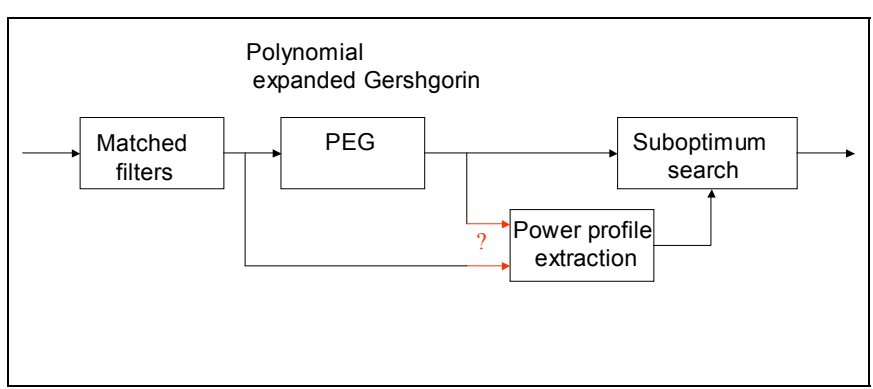

Figure 2. Power profile extraction of the users

power profile, the search will be carried out on the weakest users in the system.

The power profile calculation could be carried out in two separate places in the structure. It could be either before the initial stage of the RRLG or be after initial stage, and before the sub optimum search stage, Figure 1. Power profile extraction in the very input of the initial stage has a benefit in the actual systems, as they usually have a power control unit already in the system and these information will be ready by that subsystems without introducing any extra overhead to the system. However they are not as accurate as the one that calculates the power profile based on the output of the RRL detector. These two different approaches have been compared in the next section and the second one outperforms the first one.

Simulations in the next section show that by confining the number of weakest users to be $40 \%$ of the all users will give almost the same performance.

\section{RESULTS AND DISCUSSIONS}

Different simulations are carried out which the results are given as follow:

Fig(3) compares the performance of different RRL detectors with decorrelator and conventional detector. 2 asynchronous users using spreading codes' crosscorrelations $=0.3,0.35$, and 5 stages with three conditions: simple condition Eq.(7), modified condition Eq.(9), the RRLG Eq.(12, 13). One path fading-channel is considered. RRLG provides the best performance.

Fig(4) compares RRL detectors with the recent proposed asymptotic RRL detector in [6] in the AWGN channel. 10 
synchronous users are considered. Our detector (RRL) achieves the same performance as the actual decorrelator. In Figures 5-9, a WCDMA system, containing 10 users using processing of 16 , in Vehicular-A channel, FDDUplink is considered.

Fig(5) shows the RRLG performance operating in multiple stages. It achieves the majority of its gain in 3 stages.

Fig(6) shows the performance of the suboptimum search algorithm operating on the output of the RRLG detector introduced in section III. The detector outperforms the PPIC detector even in the low $\mathrm{Eb} / \mathrm{No}$ (in the region of 5-10 dB), which is important in the actual systems.

Performances of the search algorithm with consideration of the weakest user (smart search) is shown on Fig(7). Note that the smart search in this figure is 10 times less complex than the sub optimum normal search, as the number of users is 10.Fig(8) shows the effect of the number of the weakest users considered in the search algorithm. Only 5 users give performance almost the same as the total number of users. Confining the search process to 4 users seems satisfactory, which means $60 \%$ reduction in complexity.

Fig(9) compares the performance of the 4 weakest users obtained in two different stages:

a) SINR estimated at output of matched filters (input to initial stage of RRLG)

b) SINR estimated at output of initial stage of RRLG (input to suboptimum search algorithm)

It is well shown that estimation of the powers using scheme (b) outperforms the one estimated by (a). This behaviour becomes clearer in the high interference regimes.

The complexity evaluations of RRLG and the Fourier algorithm are depicted on Figures 10 and 11. For calculating the operations involved in the Fourier algorithm we have used the program presented in [8]. In the Fourier algorithm the FFT length of $D=32$ was considered. 12 users are considered and the maximum channel impulse response is 57 chips.

Figure 10 shows the comparison of two methods and the effect of the number of receiver antennas on the complexities. In this simulation a large value for the spreading factor is considered (128). As can be seen, for large values of spreading factor, the Fourier algorithm suffers from the complexity. As shown in the graph, this behaviour can be magnified as the number of the received antennas increases. The effect of the spreading factor is shown in Figure 11. As the spreading factor varies, the number of bits that each user transmits $\left(N_{\mathrm{b}}\right)$, varies, because for the FDD-WCDMA uplink in each slot (Spreading factor * Number of transmitted bits $N_{\mathrm{b}}$ ) $=2560$ remains the same. It can easily be seen that for large values of the spreading factor, the complexity of the Fourier algorithm is high, however in low spreading factors its complexity is much better than other schemes. In this simulation, one antenna is considered. The reason for this behaviour in the complexity can be described as follow:

As RRLG operations are performed on the output of matched filters $(\mathbf{y})$, there is no dependency on the spreading factor anymore. In the situations that large values of spreading factor are considered, this will avoid the complexity to increase a lot. On the other hand in the Fourier algorithm the main operations are carried out at the received signal and depend on the spreading factor and hence by increasing the value of spreading factor the complexity increases a lot.

\section{CONCLUSIONS}

A low complex structure composed of suboptimum search algorithm in conjunction with a reduced rank linear multiuser detector for the mobile uplink was proposed. Looking analytically on the convergence conditions of the initial stage, a modification was applied that apart from its simplicity had an improved performance. Its complexity was also compared to the recently proposed Fourier method. The suboptimum search algorithm was using a sequential method and did not have a high degree of complexity. Both search algorithm and primary stage were using similar information that avoided unnecessary extra calculations. Power profile of the users was also considered in the search algorithm and by sacrificing a little amount of performance the complexity was even more reduced. The techniques are low complex and operate on the canonical formulation of the systems, which in consequence become suitable for implementation and applicable in MC-CDMA and MIMO systems. Several simulations were carried out for evaluating the performances. They were also compared to partial parallel interference cancellation method.

\section{REFERENCES}

[1] Lei Z.D., Lim T.J., "Simplified Polynomial-Expansion linear Detectors for DS-CDMA Systems", IEE Electronic Letters, Vol. 34, No. 16, pp.1561-1563, Aug. 1998

[2] Verdu S., "Multi-user Detection", Cambridge university press, 1998

[3] Moshavi, S., Kanterakis, E.G., and Schilling, D.L.: " Multistage linear receivers for DS-CDMA systems", Int. J. Wirel. Inf. Newtw., 1996, 3, (1), pp.1-17

[4] M. Mozaffaripour, R. Tafazolli, "Fast Linear Multi-user Detector for DS-CDMA Systems", Capacity and range enhancement techniques for the third generation mobile communication and beyond, London, February 2000

[5] Helmut Lutkepohl, "Handbook of Matrices", John Wiley \& Sons Ltd, 1996

[6] R. Mueller and S. Verdú, "Design and Analysis of LowComplexity Interference Mitigation on Vector Channels", IEEE Journal on Selected Areas on Communications, vol. 19, no. 8, pp. 1429-1441, Aug. 2001

[7] M. Vollmer, M. Haardt, J. Götze, "Comparative Study of JointDetection Techniques for TD-CDMA Based Mobile Radio Systems", IEEE Journal on Selected Areas in Communications, Vol.: 19, No. 8, August 2001, pp1461-1475.

[8] M. Vollmer. Programs for computing computational requirements of four JD algorithms. [Online]. Available: http://www-dt.e-echnik.unidortmund.de/mitarbeiter/mvo/compreq.html

[9] M.Mozaffaripour, "Multiuser Detection for Mobile CDMA Systems", PhD thesis, CCSR, University of Surrey, Aug. 2003. 


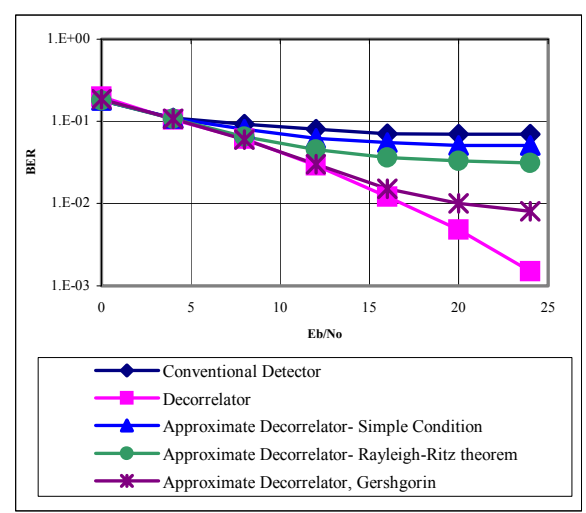

Figure 3. Decorrelator with 2 asynchronous users, Crosscorrelations $=0.3,0.35$, and 5 stages with three conditions: simple condition Eq.(7), modified condition Eq.(9), and a condition based on Gershgorin theorem Eq.(12, 13). One path fading channel

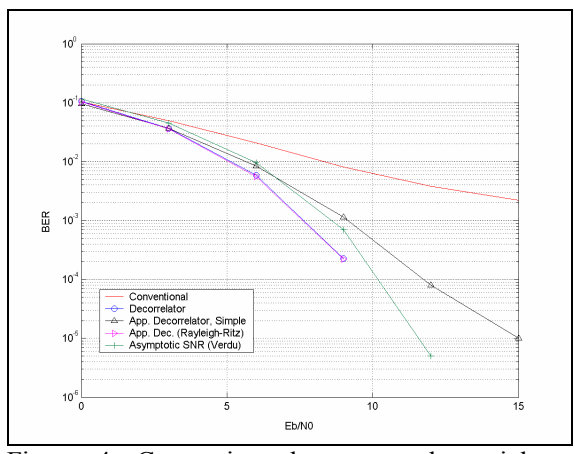

Figure 4. Comparison between polynomial expanded linear detectors implemented in three stages, 10 synchronous users, AWGN channel. Three methods for calculation of the coefficients: a) Simple method $\mathrm{Eq}(9)$, b) Rayleigh-Ritz Theorem Eq(9), c) Asymptotic SNR optimisation [6]

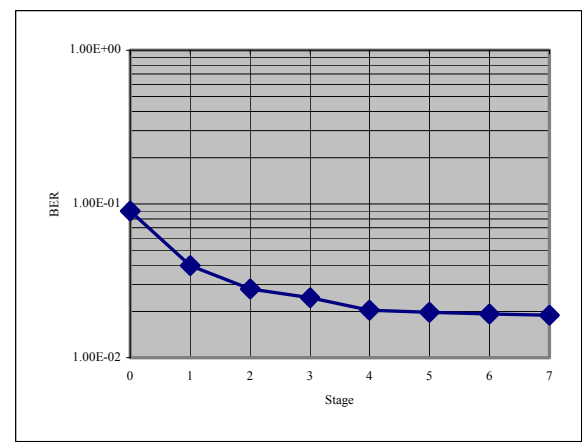

Figure 5. Multistage effect on the performance of the RRLG detector, 10 asynchronous users, Random spreading codes of length 16 ,

Vehicular A fading channel, $\mathrm{E}_{\mathrm{b}} / \mathrm{N}_{0}=14 \mathrm{~dB}$

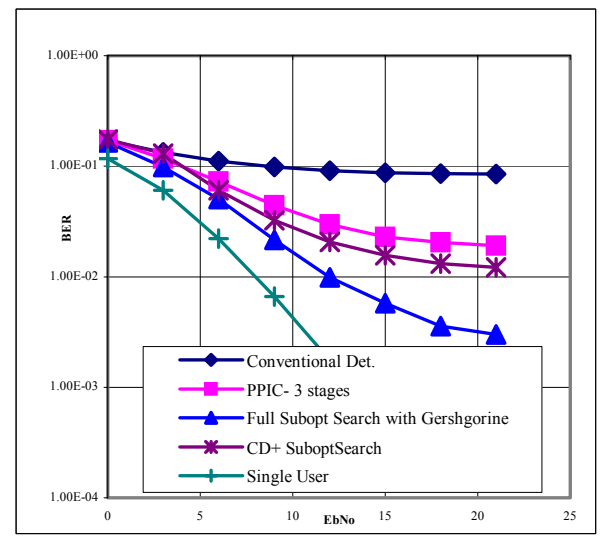

Figure 6. Performance of the Suboptimum search algorithm for 10 Users, Multipath fading environment, $\mathrm{PG}=16,3$ stages of RRLG, Vehicular A Channel

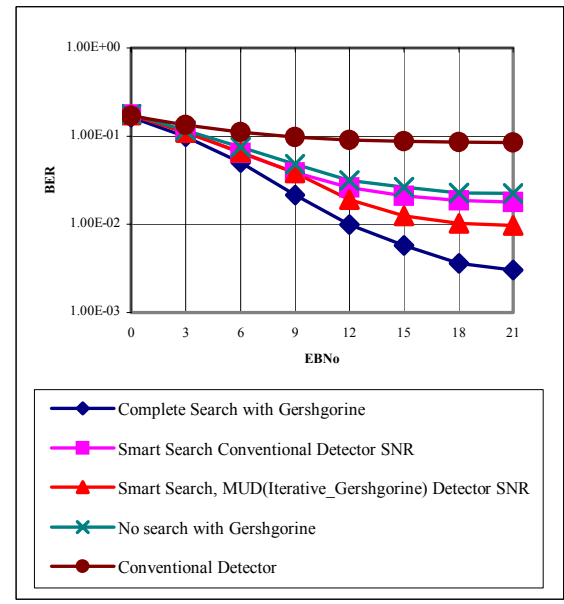

Figure 7. Performance of smart search in compare with full search in the suboptimum search algorithm for 10 Users, Multipath fading environment, $\mathrm{PG}=16,3$ stages of RRLG

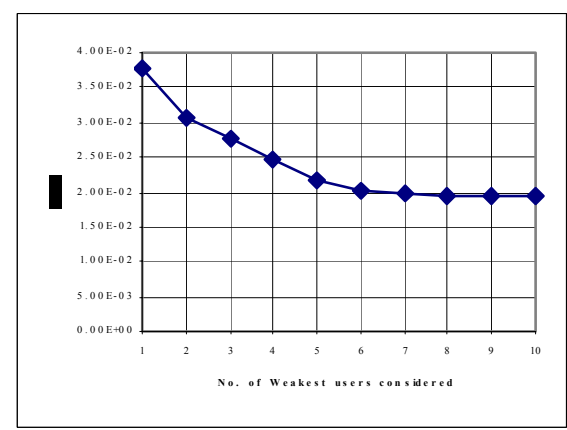

Figure 8 . Effect of number of weakest users considered in the search process, first stage is conventional detector, $\mathrm{PG}=16,10$ users transmitting at $9 \mathrm{~dB}$, Vehicular A Channel

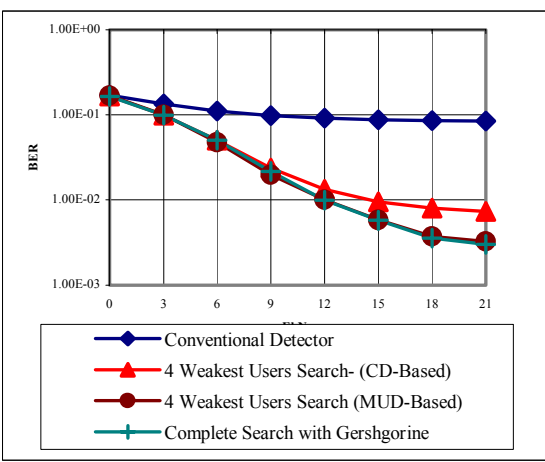

Figure 9. Different number of weakest users considered in the search algorithm. Reducing the complexity by $60 \%$ and having the performance almost the same

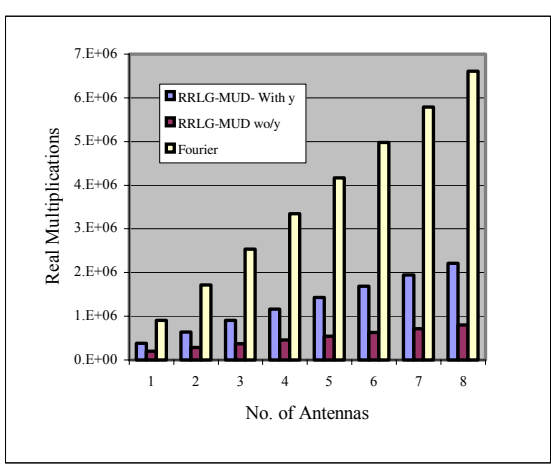

Figure 10. Effect of number of antennas on complexity. Spreading factor of users is 128 .

Number of symbols transmitted by each user is 10 , $I_{\max }=3$.

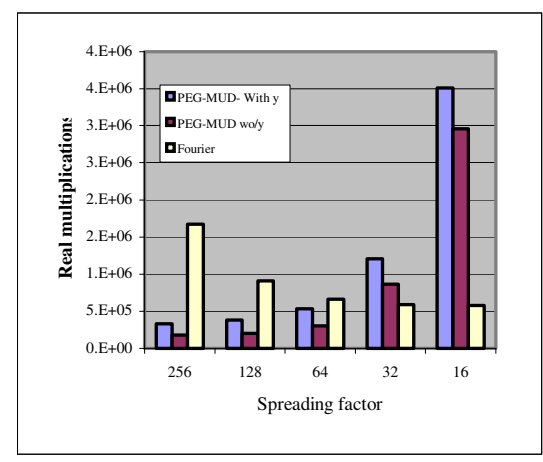

Figure 11. Effect of spreading factor on the complexity of algorithms. One antenna is considered 